\title{
Substrate Topography Regulates Differentiation of Annulus Fibrosus-derived Stem Cells via CAV1-YAP-mediated
}

\section{Mechanotransduction}

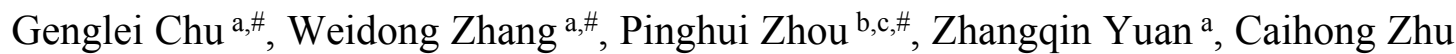

a, Huan Wang a, Jiaying Li a , Feng Zhou ${ }^{\text {a }}$, Qiang Yang d,e,*, Huilin Yang a,*, Bin Li a,e,*

a Department of Orthopaedic Surgery, Orthopaedic Institute, The First Affiliated Hospital, Medical College, Soochow University, Suzhou, Jiangsu, China;

b Department of Orthopaedic Surgery, The First Affiliated Hospital, Bengbu Medical College, Bengbu, Anhui, China;

${ }^{c}$ Anhui Province Key Laboratory of Tissue Transplantation, School of Life Sciences, Bengbu Medical College, Bengbu, Anhui, China;

d Department of Spine Surgery, Tianjin Hospital, Tianjin, China;

e China Orthopaedic Regenerative Medicine Group (CORMed), Hangzhou, Zhejiang, China.

\# These authors contributed equally to this work.

* Corresponding to:

Prof. Bin Li, 708 Renmin Rd, Rm 308 Bldg 1, Soochow University, Suzhou, Jiangsu 215007, China

E-mails: binli@suda.edu.cn (BL); yangqiang1980@126.com (QY); suzhouspine@163.com (HY)

Number of pages: 5

Number of figures: 3

Number of tables: 2 
Table S1. Sequences of primers for RT-qPCR.

\begin{tabular}{|c|c|c|c|}
\hline Gene & Forward & Reverse & $\begin{array}{l}\text { Accession } \\
\text { number }\end{array}$ \\
\hline \multirow{2}{*}{ GAPDH } & 5'-ACTTTGTGAAGCTCATTT & 5'-GTGGTTTGAGGGCTCTTACT & NM_0010 \\
\hline & CCTGGTA-3' & CCTT-3' & 82253 \\
\hline \multirow{2}{*}{ Colla1 } & 5'-CTGACTGGAAGAGCGGA & 5'-CCATGTCGCAGAAGACCTT & AY63366 \\
\hline & GAGTAC-3' & GA-3' & 3 \\
\hline Col2a1 & $\begin{array}{l}\text { 5'-AGCCACCCTCGGACTCT- } \\
3^{\prime}\end{array}$ & 5'-TTTCCTGCCTCTGCCTG-3' & $\begin{array}{l}\text { NM_0011 } \\
95671\end{array}$ \\
\hline \multirow{2}{*}{ Acan } & 5'-ATGGCTTCCACCAGTGC & 5'-CGGATGCCGTAGGTTCTCA- & XM_0027 \\
\hline & G-3' & $3^{\prime}$ & 23376 \\
\hline \multirow{2}{*}{ Adamts 17} & 5'-GGATAAGCCTGCCCACA & 5'-CCCCTTGGCGGAAATCTTCT & XM_0082 \\
\hline & GTT-3' & $-3^{\prime}$ & 48768.2 \\
\hline \multirow{2}{*}{ Sfrp2 } & 5'-TCGAGTACCAGAACATG & 5'-GAAGAGCGAGCACAGGAA & XM_0027 \\
\hline & CGG-3' & CT-3' & 16888.3 \\
\hline \multirow{2}{*}{ Col5al } & 5'-CCAGACAGAGCACGAGG & 5'-GAAGGTAAGGGAAACGCAC & XM_0173 \\
\hline & GAG-3' & AT-3' & 40399.1 \\
\hline \multirow{2}{*}{ Col12al } & 5'-TTGGGGCTTATTGGACA & 5'-GACTGTGGGTGGCACTTTG & XM_0082 \\
\hline & TCTG-3' & G-3' & 63114.2 \\
\hline \multirow{2}{*}{ Sox 9} & 5'-AAGCTCTGGAGACTTCT & 5'-CGTTCTTCACCGACTTCCTC & XM_0082 \\
\hline & GAACG-3' & $\mathrm{C}-3^{\prime}$ & 71763 \\
\hline \multirow{2}{*}{ Mkx } & 5'-GCTTGTTGAACCGCTAC & 5'-CCGTCTTGACTTCCGCTAT-3 & XM_0082 \\
\hline & CT-3' & ' & 68035 \\
\hline Den & $\begin{array}{l}5^{\prime} \text {-CCTGACACGACGCTACT- } \\
3^{\prime}\end{array}$ & 5'-TGAGGGATGGTGGTTAT-3' & $\begin{array}{l}\text { NM_0010 } \\
82330\end{array}$ \\
\hline Bgn & $\begin{array}{l}\text { 5'-TTGAGAACGGGAGCCTG } \\
\text { AG-3' }\end{array}$ & 5'-GTTGCCGAACTGGATGG-3' & $\begin{array}{l}\text { NM_0011 } \\
95691\end{array}$ \\
\hline \multirow{2}{*}{ Coll4al } & 5'-TGGATGACGGAAGTGAG & 5'-GGCTGGTCGGTTCTACAGTT & XM_0027 \\
\hline & AGTGAGG-3' & GTG-3' & 10753 \\
\hline \multirow{2}{*}{ Msx2 } & 5'-TGCAAGCGGCATCCATA & 5'-ACCGGCGTGGCATAGAGTC & XM_0027 \\
\hline & TACAGC-3' & $\mathrm{C}-3^{\prime}$ & 10390 \\
\hline
\end{tabular}


Table S2. Mechanical properties of electrospun PECUU scaffolds.

\begin{tabular}{|c|c|c|c|}
\hline Scaffold & Young's modulus ${ }^{1}(\mathrm{MPa})$ & Tensile strength ${ }^{1}(\mathrm{MPa})$ & Young's modulus ${ }^{2}(\mathrm{MPa})$ \\
\hline $\mathrm{SF}$ & $3.6 \pm 0.2$ & $2.8 \pm 0.5$ & $4.4 \pm 0.6$ \\
\hline MF & $3.7 \pm 0.4$ & $2.9 \pm 0.9$ & $4.6 \pm 0.5$ \\
\hline $\mathrm{LF}$ & $4.0 \pm 0.3$ & $3.1 \pm 0.8$ & $4.7 \pm 0.6$ \\
\hline
\end{tabular}

${ }^{1}$ Mechanical properties measured by uniaxial tensile testing. Sample thickness, $\sim 0.1 \mathrm{~mm} ; \mathrm{n}=4$.

${ }^{2}$ Young's modulus measured by nanoindentation.

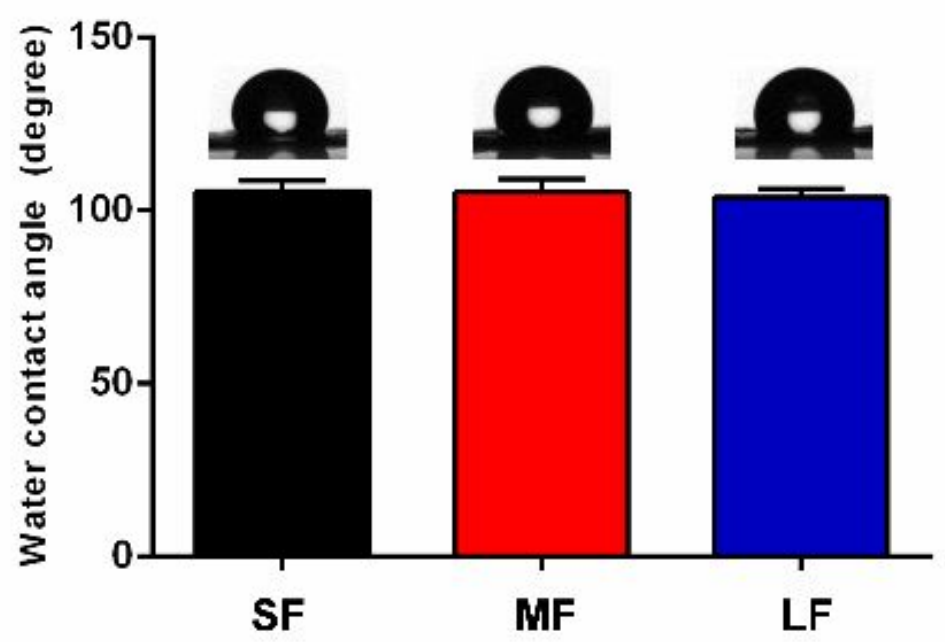

Figure S1. Water contact angle of the electrospun fibrous scaffolds. SS: soft, small fiber; FS: stiff, small fiber; SL: soft, large fiber; FL: stiff, large fiber. 

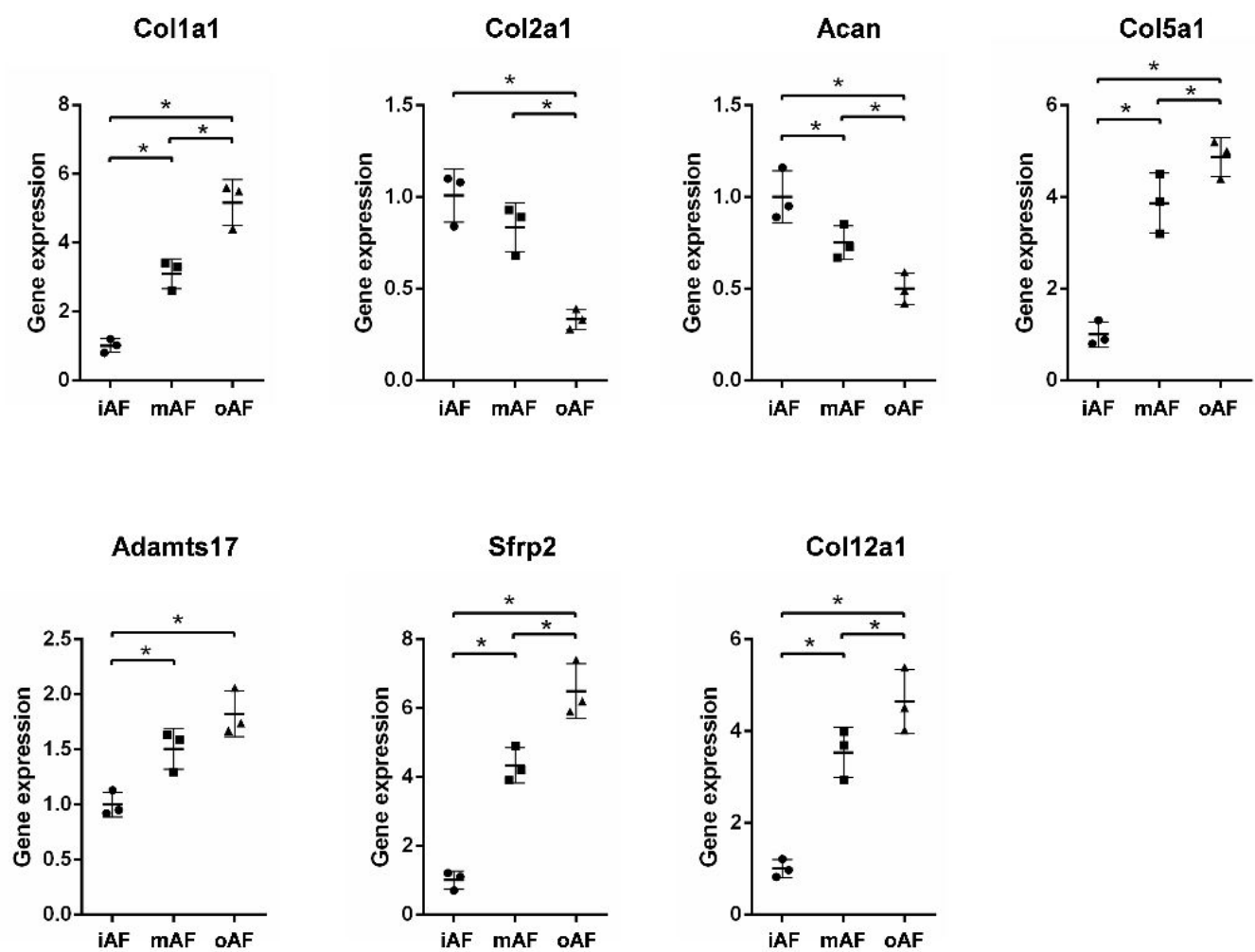

Figure S2. AF marker genes Colla1, Col2a1, Acan, Col5a1, Adamts17, Sfrp2 and Col12al discriminated oAF from iAF in cells. Gene expression was normalized to GAPDH expression. *, $p<0.05$.

A

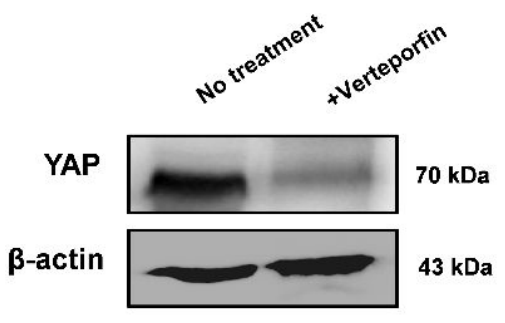

Bgn

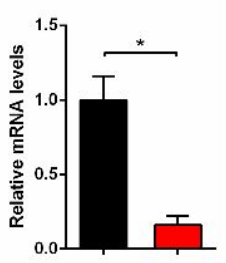

B

Den

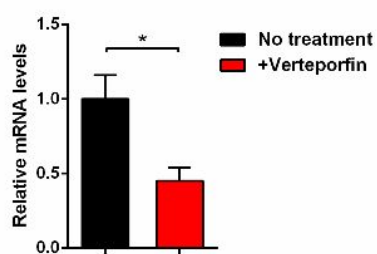

Msx2

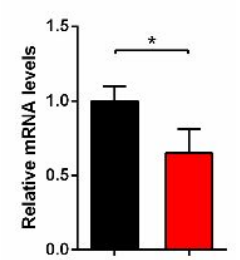

Mkx

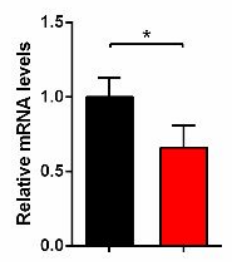

Sox-9

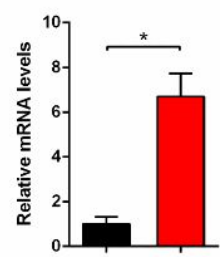

Figure S3. YAP activity drove tendon/ligament-related gene expression. (A) Western blot analysis of YAP expression level in AFSCs treated with or without verteporfin. 
(B) Relative expression of tendon/ligament makers in AFSCs after 7 days of culture with or without verteporfin. ${ }^{*}, p<0.05$. 